\title{
Understanding Enzyme Catalysis Using Computer Simulation
}

\author{
Jerry M. Parks, ${ }^{\dagger}$ Petra Imhof, ${ }^{\dagger}$ and Jeremy C. Smith ${ }^{*},, \mathbb{A}$ \\ UT/ORNL Center for Molecular Biophysics, Oak Ridge National Laboratory, 1 Bethel Valley \\ Road, Oak Ridge, Tennessee, 37831-6309, Computational Molecular Biophysics Group and \\ Computational Biophysical Chemistry Group, IWR University of Heidelberg, Im Neuenheimer \\ Feld 368, 69120 Heidelberg, Germany, and Department of Biochemistry and Cellular and \\ Molecular Biology, University of Tennessee, Knoxville, Tennessee, 37996 \\ E-mail: smithjc@ornl.gov
}

\begin{abstract}
Enzymes catalyze biochemical reactions with remarkable specificity and efficiency, usually under physiological conditions. Computer simulation is a powerful tool for understanding enzyme catalytic mechanisms, particularly in cases where standard experimental techniques may be of limited utility. Here, we present an overview of the application of computer simulation techniques to understanding enzyme catalytic mechanisms. Examples using quantum chemical methods, as well as combined quantum mechanical/classical mechanical approaches, are provided.
\end{abstract}

\footnotetext{
'Oak Ridge National Laboratory

IWR University of Heidelberg

IUniversity of Tennessee
} 


\section{Keywords}

enzyme, mechanism, quantum chemical cluster, QM/MM, free energy

\section{Introduction}

Enzymes are proteins that catalyze biochemical reactions, often with high specificity and under mild, physiological conditions. ${ }^{1,2}$ They are essential to life processes, participating, for example, in signaling, metabolism, and detoxification. Enzymes are capable of increasing reaction rates by many orders of magnitude relative to uncatalyzed reactions in solution. An often-cited example is orotidine 5'-phosphate (OMP) decarboxylase, which yields a rate enhancement $\left(\mathrm{k}_{\text {cat }} / \mathrm{k}_{\text {uncat }}\right)$ of $10^{17}$ relative to the uncatalyzed reaction in solution. ${ }^{3,4}$ Enzymes are key targets for drug discovery, and they are increasingly used in industrial processes such as bioenergy production. Thus, it is important to understand how they achieve their remarkable efficiency.

Enzymes catalyze numerous reactions including, but not limited to, oxidation, reduction, addition, elimination, phosphoryl transfer, hydrolysis, substitution, isomerization, (de)carboxylation, (de)amination, and mono- and dioxygenation. ${ }^{2}$ Cofactors such as $\mathrm{NADP}(\mathrm{H}), \mathrm{FAD}(\mathrm{H}), \mathrm{Fe}-\mathrm{S}$ clusters, heme, or metal ions may be required for activity.

Enzymes may display high specificity for their substrates, for example, binding only one stereoisomer of a chiral substrate. The catalytic power of an enzyme for its substrate derives from its enhanced binding affinity for the altered substrate in the transition state. ${ }^{5}$ In other words, enzymes bind the transition state of the reaction more tightly than the ground state.

A number of strategies are employed in enzymatic rate enhancement. Some of these strategies include acid/base catalysis, covalent catalysis, hydrogen bonding, charge transfer, electrostatic stabilization, hydrophobicity, open-shell radical chemistry, and tunneling. ${ }^{1,2}$ Among the many goals of studying enzyme catalysis is to characterize and quantify the various effects that facilitate catalysis.

Although experimental techniques such as X-ray crystallography, NMR, and other spectro- 
scopic methods are critical for studying enzyme structure, they are sometimes unable to answer questions concerning detailed catalytic mechanisms. Computational approaches allow the direct assessment and characterization of the enzyme-substrate (ES) and enzyme-product (EP) complexes, as well as transient species such as metastable intermediates and transition states. Simulation also enables enzyme reaction energetics to be dissected into individual contributions. Thus, computer simulation can provide important information that is complementary to experiments.

The purpose of this article is to give an overview of some of the more common methods used in simulating enzyme catalysis and to provide illustrative examples from our own work.

\section{Enzyme kinetics}

A basic understanding of experimental enzyme kinetics is a prerequisite for simulating enzyme reactions. Here, we provide only a brief overview of the concepts we consider to be essential for enzyme simulation studies. Interested readers should consult, e.g. Fersht, for further information. ${ }^{1}$

A simple kinetic scheme representing an enzymatic reaction under steady-state conditions is often written as

$$
\mathrm{E}+\mathrm{S} \underset{\mathrm{k}_{-1}}{\stackrel{\mathrm{k}_{1}}{\rightleftharpoons}} \mathrm{ES} \stackrel{\mathrm{k}_{c a t}}{\longrightarrow} \mathrm{EP} \longrightarrow \mathrm{E}+\mathrm{P}
$$

where E represents the enzyme, $\mathrm{S}$ is the substrate, ES is the enzyme-substrate complex, and $\mathrm{P}$ is the product; $\mathrm{k}_{1}$ and $\mathrm{k}_{-1}$ are the rates of association and dissociation of the ES complex, respectively; and $\mathrm{k}_{\text {cat }}$ is the apparent first-order rate constant, also called the turnover number. Enzyme turnover is defined as the number of moles of product produced per number of moles of enzyme per unit time, and is expressed in units of inverse time $\left(\mathrm{s}^{-1}\right)$. Catalase has one of the highest known enzymatic turnover numbers, with $\mathrm{k}_{\mathrm{cat}} \approx 10^{6} \mathrm{~s}^{-1}$.

The Michaelis constant $\left(K_{m}=\left(\mathrm{k}_{-1}+\mathrm{k}_{\mathrm{cat}}\right) / \mathrm{k}_{1}\right)$ is a measure of the concentration of substrate at which the rate of an enzymatic reaction is one half its maximum, $\mathrm{V}_{\max }$. An important quantity in enzyme kinetics is $\mathrm{k}_{\mathrm{cat}} / \mathrm{K}_{m}$, which is an apparent second-order rate constant that describes the specificity of an enzyme for a given substrate. However, $\mathrm{k}_{\mathrm{cat}} / \mathrm{K}_{m}$ is not typically computed because 
$\mathrm{K}_{m}$ is more difficult to calculate than $\mathrm{k}_{\mathrm{cat}}$.

It is common to compute relative free energies in simulations of enzyme catalysis. Computed activation free energies are usually compared to experimental values derived from $\mathrm{k}_{\text {cat }} \mathrm{using}$ Eyring Transition State Theory. ${ }^{6}$ Transition State Theory provides a connection between $\mathrm{k}_{\mathrm{cat}}$ and the free energy of activation, $\Delta G^{\ddagger}$ :

$$
k_{\text {cat }}=\kappa \frac{k_{B} \mathrm{~T}}{h} \exp \left[-\Delta \mathrm{G}^{\ddagger} / \mathrm{RT}\right]
$$

where $\kappa$ is the transmission coefficient (usually set equal to 1), $k_{B}$ is Boltzmann's constant, $T$ is the temperature in Kelvin, $h$ is Planck's constant, and $R$ is the gas constant expressed in $\mathrm{kcal} / \mathrm{mol}$. Transition State Theory is expected to be a good approximation for energy barriers above $\sim 10$ $\mathrm{kcal} / \mathrm{mol}$, which includes essentially all enzyme-catalyzed reactions. ${ }^{7}$

A plot of the rate of reaction as a function of $\mathrm{pH}$, or $\mathrm{pH}$-rate profile, provides valuable information for understanding $\mathrm{pH}$-dependence in enzyme-catalyzed reactions. The reaction rate may be expressed in terms or $k_{\text {cat }}, k_{\text {cat }} / K_{m}$, or both. A pH-rate profile reveals the $\mathrm{pH}$ at which an enzyme has maximal activity, as well as the number of ionizable residues that participate in the rate-limiting step of a reaction. For example, a so-called bell-shaped $\mathrm{pH}$-rate profile with a slope of +1 on its acidic side and -1 on its basic side indicates that one residue serves as a base and the other as an acid, and they must both be in the proper ionization state for activity. Apparent $\mathrm{p} K_{a} \mathrm{~s}$ for the ionizable residues may be obtained by fitting to an appropriate rate equation.

Site-directed mutagenesis is an indispensible technique for determining the effect of substituting a specific amino acid with another. For example, enzyme reaction rates can be measured for both wild-type and mutant enzymes, and changes in enzyme kinetics can be monitored to assess the possible catalytic role of a given residue. The complete absence of activity in a mutant enzyme indicates that the mutated residue is essential for catalysis.

Isotope effect measurement is an important experimental technique aiding in the determination of enzyme mechanisms. ${ }^{8}$ Mass differences between isotopes manifest themselves in equilibrium constants and reaction rates. Kinetic isotope effects (KIEs) provide information about the amount 
of bonding between important atoms or groups during the rate-limiting step of a reaction. ${ }^{9,10}$ For example, a proton transfer will proceed more rapidly than an analogous deuteron transfer, and the kinetics of the process is detectable if the process is partially or fully rate-limiting. Isotope effects are designated as primary, in which a bond involving an isotopically labeled atom is either broken or formed, or secondary, in which the isotopically labeled atom is remote from the site that undergoes a change in bond order. Isotope effects are also classified as normal $\left(\frac{k_{H}}{k_{D}}>1\right)$, or inverse $\left(\frac{k_{H}}{k_{D}}<1\right)$. KIEs of enzyme reactions are typically expressed as ${ }^{m}(V / K)$, where $m$ is the mass of the heavier isotope of the labeled atom, $V$ is the maximal rate of the reaction, and $K$ is the Michaelis constant.

\section{Computer simulation of enzyme systems}

One of the main benefits of computer simulation is that it provides information that is often complementary to experiment. Simulation can provide molecular structures at atomic detail, reaction energetics, and numerous ways to analyze the data. For example, energies obtained from a molecular simulation may be decomposed into individual components such as per-residue electrostatic contributions to catalysis. Both classical and quantum chemical methods are described in a recent book. ${ }^{11}$

Molecular mechanics is a standard tool for simulating the dynamic properties of biomolecules, including conformational changes and thermodynamic quantities. In molecular mechanics, there is no explicit inclusion of the electronic degrees of freedom. Instead, harmonic or higher-order potential terms are used to describe bonded interactions (bond distances, bond angles and dihedral angles), and atom-centered Coulombic and van der Waals potentials are used to describe nonbonded interactions. Several molecular mechanics, or force field, potentials ${ }^{12}$ have been developed, with AMBER,${ }^{13}$ CHARMM,${ }^{14}$ GROMOS, ${ }^{15}$ and OPLS ${ }^{16}$ being among the more popular. Molecular dynamics simulation (MD) involves propagating a system in time according to Newton's equations of motion and enables efficient statistical sampling of phase space for dynamical processes 
on timescales in the range of $\sim 10^{-12}$ to $10^{-6} \mathrm{~s}$. MD is the most commonly applied method, but Monte Carlo approaches are also frequently used and may provide unique advantages over MD in some instances. Polarizable force fields ${ }^{12}$ such as AMOEBA ${ }^{17}$ and SIBFA $^{18}$ allow explicit charge polarization of MM atoms, and may well be very useful in classical and combined quantum mechanical and classical simulations. Although the standard, harmonic potential form of the bonded parameters is sufficient to describe chemical bonds near their equilibrium distances, the major limitation of standard MM force fields lies in their inability to describe bond breaking and forming in chemical reactions.

Quantum chemistry is an enormous field of study that consists of three main approaches: ab initio, ${ }^{19,20}$ density functional theory (DFT), ${ }^{21,22}$ and semi-empirical quantum mechanical (SQM) methods ${ }^{23-25}$ The main strength of quantum chemistry lies in its ability to describe chemical structures, energetics, and reactions quantitatively.

\section{Quantum chemical cluster approach}

Although enzymes may consist of hundreds or even thousands of amino acids, and many of these may comprise the binding site, in general only two or three residues are directly involved in catalysis. $^{2}$ In favorable cases it is possible to describe enzyme reactions accurately by modeling only a few residues, typically between 30 and 200 atoms. ${ }^{26}$ A system of this type would consist of the substrate, any required cofactors, the residues that participate directly in the reaction, and possibly one or more explicit solvent molecules. The quantum chemical cluster approach has been the subject of several informative reviews over the past decade. ${ }^{26-35}$

The basic procedure for the cluster approach is as follows. A model of an enzyme active site is constructed by extracting the coordinates of a few important residues from an available X-ray crystal structure. The entire system is described using a high-accuracy quantum chemical method, most often with a hybrid DFT method such as B3LYP. ${ }^{36-38}$ The rest of the enzyme is not included explicitly, but is modeled as a polarizable dielectric cavity using a continuum approach ${ }^{39}$ such 
as the COSMO Polarized Continuum Method (C-PCM). ${ }^{40-43}$ A dielectric constant of $\varepsilon=4$ is frequently used, although this value may be adjusted to reflect the polarity of the active site. Geometry optimizations of stationary points (energetic minima and maxima) are performed, and a series of possible reaction paths are constructed. Often the geometries are optimized in the gas phase and solvation corrections are added later, although care must be taken to ensure that the structures and properties of the cluster are not adversely affected by this approximation. Methods for calculating continuous, analytic energy derivatives within the PCM framework have been developed, ${ }^{44}$ resulting in significant improvements over other approaches for performing geometry optimizations and calculating molecular properties. It is also common to compute the single-point energies of each structure using a higher level of theory and/or larger basis set. Zero-point energy corrections may be included to improve the accuracy of the calculations. The final energies are then compiled to construct energetic profiles for possible pathways, which are then compared with each other and with available experimental data to determine the most likely reaction mechanism.

Errors should be carefully assessed and understood for a given problem. For currently used hybrid DFT functionals, geometries are generally quite accurate, and energetic errors are typically in the range of 3-5 kcal/mol. ${ }^{45-47}$ Thus, results are often of sufficient accuracy to distinguish between likely and unlikely mechanisms and to assist in interpreting experimental data. One method for ensuring the convergence of results with respect to system size in quantum chemical cluster calculations is known as "accretion", 26,31,35,48 which involves systematically increasing the number of atoms in the cluster until PCM solvation corrections no longer significantly affect the result.

\section{Combined QM/MM methods}

Enzymes are large biomolecules consisting of thousands of atoms. However, the active site may comprise only $\sim 100$ atoms. While quantum chemical calculations are nowadays affordable for up to a few hundred atoms (depending on the level of accuracy), solving the Schrödinger equation for an entire enzyme system is, in general, not feasible. In addition to the quantum cluster approach, 
another popular method is to describe the "region of interest" of an enzyme at the most accurate level of theory possible while taking into account the surrounding protein environment (and perhaps the solvent) with a molecular mechanics potential. This is the essence of the combined quantum mechanical/molecular mechanical (QM/MM) method. ${ }^{49-51}$

Because of the number of different quantum chemical and molecular mechanical programs that exist, there are countless conceivable combinations of QM and MM programs. As such, there are nearly as many different "flavors", or implementations, of QM/MM as there are research groups performing such calculations. A number of reviews have been written on various aspects of QM/MM simulation, ${ }^{51-63}$ the most comprehensive of which being two recent reviews by Senn and Thiel. ${ }^{64,65}$

\subsection{The QM/MM boundary}

One aspect of QM/MM simulation that requires special attention is the interface between the quantum mechanical and classical regions. Here, we refer to both the physical location of the boundary (the size of the regions described at the different levels) and how the two regions interact with each other across the boundary.

For a small-molecule solute in solution, a straightforward choice for partitioning the two regions is to describe the solute quantum mechanically and use molecular mechanics for the solvent. The interaction between the two regions is then purely "non-bonded". That is, the interaction between the solute and solvent is limited to the electrostatic and van der Waals forces between non-covalently bound atoms.

In other situations, when modeling larger enzymatic systems within a QM/MM framework, the choice of the quantum mechanical region is less obvious. For reasons of computational cost, the QM region should be as small as possible. A minimal QM region would typically include all atoms that undergo a change in the number and/or type of bonds during the reaction, either by bond breaking or forming, or by a change in hybridization, as in the transformation from a single to a double bond. As a simple rule, those atoms that would otherwise require a change in atom type 
in an empirical force field must be included in the quantum mechanical subsystem. In addition, groups for which a significant change in electron density is anticipated cannot easily be described properly by an MM force field and may be included in the QM region. Alternatively, polarizable force fields $12,17,18$ are likely to provide improvements in this regard. The effects of including or excluding a particular group of atoms on properties that are strongly influenced by an electrostatic field, such as $\mathrm{p} K_{a}$ values, must be properly examined and evaluated. In other words, the QM/MM boundary should be chosen so that it is far enough away from the reactive center to avoid artifactual effects.

In many enzymes, a substantial part of the protein itself plays a significant role in the chemical reaction. Along with the substrate, any required cofactors, and sometimes active-site water molecules, certain amino acid residues must be described quantum mechanically. Inclusion of residues in the $\mathrm{QM}$ subsystem requires one or more covalent bonds across the $\mathrm{QM} / \mathrm{MM}$ boundary. The bond between the $\mathrm{C}_{\alpha}$ and $\mathrm{C}_{\beta}$ atoms of an amino acid is a popular choice for the $\mathrm{QM} / \mathrm{MM}$ interface, as it is a reasonably well behaved, nonpolar bond, and it is often sufficiently far away from the reactive center.

Partitioning a single bond into two parts involves dividing an electron pair, usually with the QM boundary atom left in an open-shell configuration. Various schemes have been developed to tackle this problem. A straightforward approach is to cap the unsaturated atom with a dummy or so-called "link atom", usually a hydrogen. To avoid steric and electrostatic conflicts with the MM subsystem, some bonded parameters and partial charges of nearby MM atoms must be modified.

In a semi-empirical framework, the inclusion of extra hydrogen atoms can be avoided through the use of the Adjusted Connection Atom (ACA) approach, in which a boundary atom is parameterized to mimic a methyl group. ${ }^{66}$ Alternative approaches do not involve addition or change of atom types, but a special treatment of the electronic orbitals is employed for the QM/MM boundary atoms. In the local frozen orbital approach, ${ }^{49,67}$ one singly occupied orbital is defined to be along the truncated bond that is neglected (i.e., frozen) in the wavefunction calculation. It has been found that if carried out carefully, the local orbital treatment and the link atom approach are of similar 
accuracy. ${ }^{68}$

A similar concept is used in the generalized hybrid-orbital (GHO) method. ${ }^{69-71}$ In this approach, the QM subsystem is augmented by an additional atom at the QM/MM boundary. All of the orbitals of this atom remain frozen except the one forming the boundary bond.

Another QM/MM boundary approach is the pseudobond method, ${ }^{72-75}$ in which the molecular mechanical boundary atom is replaced by a quantum mechanical, fluorine-like atom with a parameterized effective core potential (ECP) and basis set. This atom has 7 valence electrons, and hence a single free valence with which to form a bond with the rest of the QM subsystem. The ECP and basis set parameters are designed to make the bond to the boundary atom behave like the bond being replaced (for example, a $\mathrm{C}\left(\mathrm{sp}^{3}\right)-\mathrm{C}\left(\mathrm{sp}^{3}\right)$ or $\mathrm{C}\left(\mathrm{sp}^{3}\right)-\mathrm{N}\left(\mathrm{sp}^{3}\right)$ bond), and to minimize the perturbation on the rest of the QM subsystem. The main advantages of the pseudobond method are that no additional degrees of freedom are introduced into the system (as in the link atom approach), the parameters are transferable to various DFT and ab initio methods, no additional programming is required when used with standard quantum chemistry software, and the method is independent of the molecular mechanics force field.

Instead of atom-centered basis functions, plane waves are used in conjunction with Car-Parrinello molecular dynamics,${ }^{76}$ and pseudopotentials are applied to avoid the explicit calculation of core electron effects. For combined QM/MM calculations, the pseudopotential of the boundary atom is specially optimized so that it minimizes errors in the electronic structure arising from truncation of the QM region.

\subsection{Modeling solvation and electrostatic effects}

Enzyme reactions usually take place in aqueous solution. Consequently, the solvent must be taken into account in modeling such reactions. The most straightforward way to do this is through the explicit inclusion of a number of water molecules in the model. One of the most commonly used molecular mechanical water models is TIP3P, ${ }^{77}$ although others such as $\mathrm{SPC}^{78}$ and $\mathrm{SPC} / \mathrm{E}^{79}$ are also used. In order to mimic the behavior of the bulk solvent, the enzyme is "immersed" in either a 
box or a spherical "droplet" of water. A solvent box has the advantage that it is able to mimic bulk effects through the application of periodic boundary conditions.

One of the most critical aspects of QM/MM simulation is an accurate, balanced description of electrostatic effects. For large systems, the explicit calculation of all charge-charge interactions is computationally quite expensive, but cutoff-based electrostatics schemes neglect important long-range effects. A reliable and efficient treatment for describing the long-range characteristics of electrostatic interactions in simulations performed under periodic boundary conditions is the Particle-Mesh Ewald method, ${ }^{80}$ which has recently become popular in the framework of QM/MM calculations. ${ }^{81,82}$ Alternatively, a system of interest may be surrounded by a sphere of explicit solvent molecules and subjected to stochastic boundary conditions. ${ }^{83-85}$ The Generalized Solvent Boundary Potential (GSBP) method ${ }^{86-90}$ has been found to be quite accurate and useful in nonperiodic QM/MM simulations of enzymes.

A simple, efficient approach for modeling electrostatic screening by the solvent is non-uniform charge scaling (NUCS). ${ }^{91}$ In the NUCS approach, the charges of the solute atoms are scaled so that the vacuum-calculated interaction energies reproduce the interaction energies computed for unscaled but shielded charges in solution. Other charge scaling procedures combining explicit and implicit solvation have also been developed. ${ }^{92,93}$ In the Variational Electrostatic Projection method, ${ }^{94,95}$ the solvent-solute boundary surface is discretized and electrostatic interactions are expanded in terms of Gaussian surface elements. Mixed explicit/implicit solvent approaches, in which only a few solvent molecules are treated explicitly, have also been used. ${ }^{96,97}$ Other QM/MM electrostatics methods have been reviewed comprehensively by Senn and Thiel. ${ }^{64,65}$

\subsection{Kinetic isotope effects}

Kinetic isotope effects result from the mass-dependence on the zero-point energy and the contributions from the translational, rotational and vibrational partition functions. ${ }^{11}$ Thus, rate constants and corresponding activation free energies exhibit different values when various isotopes 
are present in a system. In the case of hydrogen transfer reactions, tunneling effects may be important because of the light mass of the hydrogen nucleus. ${ }^{98}$ One approach for including nuclear quantum effects is the quantized classical path (QCP) approach, ${ }^{99}$ which uses a Feynman path integral formulation to calculate deuterium isotope effects. In the context of QM/MM calculations, ensemble-averaged variational transition-state theory with multidimensional tunneling (EAVTST/MT) has been used to determine tunneling contributions to lowering free energy barriers in enzyme-catalyzed reactions. ${ }^{100,101}$ Another innovative QM/MM approach for including tunneling effects involves treating the catalytic hydrogen nucleus as a vibrational wavefunction while all other nuclei are described classically. ${ }^{102,103}$

\subsection{Minimum-Energy Reaction Paths}

Reaction pathways for enzyme-catalyzed reactions can be obtained in many different ways. All methods begin with one or more energy-minimized structures corresponding to the end state(s). A straightforward approach is to define a reaction coordinate and then "drive" the system from the reactant state, over the transition state barrier, to the product state in several small steps. For a simple reaction involving the breaking of one bond and formation of another, the reaction coordinate might be defined as the difference in the length of the bond to be broken and the one to be formed. The reaction coordinate is then constrained to a specific value while all other degrees of freedom are optimized. Upon convergence the reaction is incremented; this process is repeated until the reaction is driven to completion at the product state. The reaction coordinate driving approach provides only an estimate of the actual reaction pathway and may in some cases fail completely. ${ }^{104}$ To overcome this shortcoming, several methods have been developed to determine pathways between a pair of given end states. Examples include the Self-Penalty Walk method, ${ }^{105,106}$ the Nudged Elastic Band (NEB) method, ${ }^{107}$ the Conjugate Peak Refinement (CPR) method, ${ }^{108}$ and the Quadratic String Method (QSM). ${ }^{109}$ In each of these "chain-of-states" methods, the reaction path is optimized beginning with an initial guess or interpolated path. The number of states can be constant (NEB and QSM) or dynamic (CPR). Usually only an approximation of the true minimum energy path is obtained, but the optimized pathway provides a "trajectory" of configurations along the re- 
action coordinate. Approximate transition states can be further optimized to true first-order saddle points if higher accuracy is desired.

An alternative approach to chain-of-states methods is the Discrete Path Sampling method. ${ }^{110}$ This method explores the potential energy landscape by optimizing local minima and transition states and connecting them to give different reaction pathways leading from the reactant state to the product state. In the same spirit, the Transition Network approach ${ }^{111}$ allows uniform sampling of energy minima and transitions connecting them. Determination of the most probable reaction pathway and several alternative mechanisms is performed simultaneously.

\subsection{Potential of Mean Force/Free Energy Calculations}

Minimum (potential) energy pathways can in favorable cases provide a reasonably accurate picture of the chemical processes in enzymatic reactions, specifically for enthalpically driven processes. However, it is desirable, and often necessary, to compute free energy pathways to understand these complex processes in more realistic detail. Free energy simulations based on statistical sampling can help determine, or provide reasonable estimates of, the free energy changes associated with a chemical reaction. The free energy change along a given reaction coordinate is called the potential of mean force (PMF), and can in principle be generated from molecular dynamics simulations (assuming quantum mechanics is used). However, especially when high-barrier processes are considered, direct simulation will result in getting stuck in the low energy regimes of the end states and it is unlikely that the transition of interest would ever be observed in the limited simulation time. Several approaches have been developed in order to address the sampling problem, such as using biasing potentials or reducing the phase space to sample the relevant degrees of freedom. ${ }^{112,113}$ Comparisons of various approaches for calculating QM/MM free energies can be found in e.g., refs $62,64,65,114,115$. Only some of the most common approaches are described here. 


\subsubsection{Umbrella Sampling}

The key idea of the Umbrella Sampling method ${ }^{116}$ is to modify the potential energy function so that otherwise unfavorable states are sampled sufficiently. Similar to the constraints imposed in the Reaction Coordinate Driving approach, a restraint or biasing potential is applied to force the system to sample high-energy regions of the reaction path. The reaction path is split into several (perhaps 10-20) sequential intervals or windows along the desired reaction coorinate, and (non-Boltzmann) molecular dynamics or Monte Carlo simulations are performed for each window. The bias is subsequently removed to obtain the free energy profile, using for example, the Weighted Histogram Analysis Method (WHAM). ${ }^{117}$ Another promising new method for obtaining free energy profiles is the Multistate Bennett Acceptance Ratio (MBAR) estimator. ${ }^{118}$ An ideal biasing potential would be the negative of the potential of mean force, which is not known a priori. This problem is tackled in adaptive Umbrella Sampling methods, in which an updating algorithm is applied to adjust the biasing potential on the fly. ${ }^{119,120}$ Because molecular dynamics is involved, umbrella sampling is generally limited to QM/MM simulations in which the QM subsystem is described using SQM methods.

\subsubsection{Free Energy Perturbation}

Free energy perturbation (FEP) involves describing a target system as a reference system plus a perturbation. ${ }^{121}$ The free energy differnce between two systems is calculated as the ensemble average of the perturation, sampled over the reference system. Calculation of free energies for chemical reactions is carried out by calculating free energy changes between points along a reaction pathway, which then sum up to the total free energy difference. ${ }^{122}$ In QM/MM simulations, the reference system consists of the full QM/MM system with the QM held at fixed positions, and the perturbation is the geometric displacement of the QM subsystem from point $n$ to point $n+1$ along the reaction pathway. Dynamic sampling is then carried out only for the MM atoms. ${ }^{123}$ To increase the sampling efficiency, the electron density of the QM subsystem can be approximated by

partial charges obtained from fitting to the molecular electrostatic potential (ESP charges). ${ }^{124-126}$ 
The FEP method requires the definition of the reaction pathway on the potential energy surface, as in the minimum energy pathway methods described above.

The QM/MM Minimum Free Energy Path (QM/MM-MFEP) method ${ }^{122,127,128}$ is a free-energy extension to the QM/MM Free Energy Perturbation (QM/MM-FEP) method. ${ }^{123}$ In the QM/MMMFEP method, a sequential sampling and optimization procedure is employed in which molecular dynamics simulations are performed for the MM subsystem and geometry optimizations are performed for the QM subsystem. The QM subsystem is optimized on the PMF surface for the system obtained during molecular dynamics simulations. Upon completion of the QM optimization, additional sampling is performed with the improved QM geometry and charges. The process is repeated until convergence. As in other reaction path methods, a chain of replicas is generated and subsequently optimized to obtain the final reaction path. Free energy perturbation is performed onthe-fly to obtain the PMF for the entire reaction path. The major strengths of the QM/MM-MFEP method are the well-defined PMF surface for efficient geometry optimization, and the elimination of the dependence of reaction paths on the initial geometries.

\subsubsection{Metadynamics}

Metadynamics and related approaches such as the Local Elevation Method ${ }^{129}$ and Conformational Flooding ${ }^{130}$ enable systems to escape free energy minima in MD simulations. ${ }^{131-133}$ Instead of calculating free energy changes along a single reaction coordinate, in metadynamics the free energy surface is explored as a function of a few collective variables. In a history-dependent manner, the explored surface is "filled" with repulsive Gaussian potentials to prevent the system from visiting regions that have already been explored. The resulting sum of all Gaussian terms is the negative of the free energy surface.

\subsubsection{Transition Path Sampling}

Transition path sampling is an iterative simulation scheme which, starting from an initial first trajectory connecting reactant and product states, additional trajectories, i.e. reaction pathways, ${ }^{134,135}$ 
are generated. A snapshot is taken from the initial pathway and its geometry is changed slightly in a Monte Carlo step. This configuration is then propagated forward and backward in time, and the resulting trajectory is checked to see if the transition of interest has taken place. When a successful pathway is obtained, further trajectories are generated starting from this new one, so as to produce a manifold of pathways. This method does not rely on the predefinition of a reaction coordinate but requires a reasonable descriptor for the transition of interest in order to judge whether a trajectory is productive or not.

\section{A brief guide to enzyme simulation}

At the beginning of a modeling study, one should first decide upon the specific catalytic process to model. This decision may sound simple, but it includes the nontrivial task of exhaustively searching the literature to determine what is already known about the system (or a related one), either from experiments or from previous modeling studies, and what questions remain to be answered. The three-dimensional structure of the enzyme, preferably with a bound substrate analog, reaction product or inhibitor, is among the most critical sources of information. Kinetics studies provide information about reaction mechanisms by revealing, for example, the $\mathrm{pH}$ dependence of the reaction, the number of elementary steps in the reaction, the rate acceleration by the enzyme, and the turnover number. Considerable kinetic isotope effects indicate a catalytically important role of the radiolabeled atoms in the reaction. Site-directed mutagenesis can confirm the importance of specific amino acid residues. Reaction mechanisms may have already been proposed in the literature, and thus provide a logical starting point for modeling studies.

Depending on whether or not the protein environment is anticipated to play a crucial role in catalysis, either an active site QM cluster model or an all-enzyme QM/MM protocol-or both-is chosen. In both cases, the QM level of theory must be chosen appropriately. In the case of an all-enzyme model a computationally less demanding, and potentially less accurate, method may have to be applied. Because of the large number of degrees of freedom in a QM/MM system, 
geometry optimizations are more difficult, and dynamic sampling may be required to prevent it from becoming trapped in local minima and to provide meaningful statistics.

In QM/MM simulations, semi-empirical methods ${ }^{23}$ such as AM1, ${ }^{136} \mathrm{OMx},{ }^{137-140} \mathrm{PM} 3,{ }^{141}$ PM6, ${ }^{142}$ PDDG/PM3 ${ }^{143-145}$ and the DFT-based SCC-DFTB approach ${ }^{146-149}$ are widely used. These methods are computationally efficient, enabling extensive statistical sampling. Among the more accurate of the SQM methods, PDDG/PM3 was recently compared to dispersion-corrected B3LYP for computing heats of formation and isomerization energies and was found to perform quite similarly and at a fraction of the computational cost. ${ }^{150}$ Other SQM methods, tuned for the system under investigation using specific reaction parameterization, ${ }^{151}$ can also reach high accuracy, as in the case of AM1/d ${ }^{152,153}$ and other Hamiltonians. ${ }^{154}$ Another promising method for improving accuracy is to augment standard SQM methods with empirical correction terms for dispersion, hydrogen bonding, or both. ${ }^{155,156}$ Recent work aimed at improving SCC-DFTB methods includes extending the DFT energy expansion from second to third order and improving the Coulomb term, ${ }^{157}$ and a parameterization for improving the description of phosphate hydrolysis reactions. 158

Because of its relative efficiency and accuracy, DFT remains popular for modeling systems up to a few hundred atoms. ${ }^{65}$ Correlated ab initio methods are also used, and may offer higher accuracy than DFT methods. ${ }^{159}$ The choice of the QM method will of course also be influenced by the size of the QM system. As stated above, the QM subsystem should be selected with care, comprising all reactive groups.

The actual enzyme model is then built starting from an available X-ray (or NMR) structure. Construction of the initial enzyme-substrate complex may require in silico back-mutation of some residues, and/or replacement of an inhibitor by the actual substrate. Missing heavy atoms and hydrogen atoms absent from X-ray structures must be added. With the addition of the hydrogen atoms comes the choice of the protonation state for titratable groups. Whereas most amino acids are predominantly in their standard protonation states (i.e. the side chain of Glu is a carboxylate, Lys is an ammonium, and Ser is a hydroxyl), the local environment of the enzyme active site 
is likely to induce $\mathrm{p} K_{a}$ shifts that result in nonstandard protonation states that are relevant for

catalysis. Special care must be taken for systems with nearby metal ions, which can significantly influence the proton affinities of surrounding amino acids. After inclusion of the solvent, either explicit or implicit, energy minimization of the system, possibly followed by a short molecular dynamics simulation, must be performed to remove any local strain. Snapshots from molecular dynamics trajectories are sometimes used to generate different initial geometries for subsequent modeling of reaction pathways.

From the computation of different reaction pathways, potential or free energies for various mechanisms are compared with each other and the most likely mechanism is identified based on energetic considerations. Comparison with experimental data is then used to substantiate or invalidate the proposed mechanism, as it has to agree with and/or explain experimental findings such as the importance of a certain residue, the dependence of a metal cofactor, the preference for certain substrates, etc. Once a likely mechanism is determined, the structural and energetic contributions to catalysis are determined using a variety of methods.

In the following sections, some of the approaches and applications described in this article are illustrated using three case studies from our own work. We begin with a mechanistic study of the bacterial organomercurial lyase, MerB, using a quantum chemical cluster method, followed by a QM/MM free energy simulation of the dual-specificity phosphatase, Cdc25B, and a dual QM and QM/MM study of the restriction enzyme EcoRV.

\section{Case study 1: Organomercurial lyase, MerB}

In recent work, the mechanism of $\mathrm{Hg}-\mathrm{C}$ bond cleavage by the bacterial enzyme organomercurial lyase (MerB) was examined using a quantum chemical cluster model. ${ }^{160}$

Mercury is toxic to living organisms and has no known biological function. Organomercurial species such as methylmercury, $\left[\mathrm{CH}_{3} \mathrm{Hg}(\mathrm{II})\right]^{+}$, bioaccumulate in living organisms due to their high affinity for thiols and other functional groups in vivo. The bacterial enzyme organomercu- 
rial lyase (MerB) catalyzes a key step in degrading toxic mercury-containing compounds, specifically by cleaving mercury-carbon bonds in methylmercury and other organomercurials (Scheme Scheme 1).

The quantum chemical cluster approach was used to study the $\mathrm{Hg}-\mathrm{C}$ cleavage, or protonolysis, reaction catalyzed by MerB in ref 160. In particular, the aim was to determine which, if any, of the mechanisms proposed previously in the literature was correct, and also to determine how MerB achieves its rate enhancement. Two previously proposed mechanisms were considered in the calculations. In both mechanisms, a covalent Cys-methylmercury adduct was designated as the reactant state, and only the $\mathrm{Hg}-\mathrm{C}$ cleavage step was considered in the calculations. In the first mechanism, one of the Cys residues in the active site provides a proton directly to the hydrocarbon leaving group. In the second mechanism, Asp99 serves as a proton shuttle to deliver the catalytic proton from Cys to the leaving group.

Included in the active site model were the methylmercury substrate, the side chains of three residues, and a single explicit water molecule (Figure 1). Using site-directed mutagenesis experiments, these three residues, Cys96 and Cys159, ${ }^{161}$ and Asp99 (S. Miller, unpublished data), were previously shown to be required for catalysis. The $\mathrm{C}_{\alpha}$ atoms were constrained to their crystallographic positions to maintain the correct active site geometry. Hybrid DFT calculations were performed for a number of possible reactant, intermediate, transition state, and product structures, and several reaction paths were constructed.

The computed activation energy for the direct proton transfer mechanism was $35.9 \mathrm{kcal} / \mathrm{mol}$, significantly higher than the experimental free energy of activation of $20.1 \mathrm{kcal} / \mathrm{mol}$ obtained using TST. In contrast, the computed activation energies for the Asp99-mediated proton transfer mechanism (20.4 or $22.4 \mathrm{kcal} / \mathrm{mol}$, depending on which Cys residue was chosen to form the initial covalent adduct with methylmercury) were both found to be within about $2 \mathrm{kcal} / \mathrm{mol}$ of the experimental value. Because experimental kinetics data is available for several organomercurial substrates, ${ }^{162}$ similar calculations were performed for two additional substrates, vinylmercury and cis-2-butenylmercury. Again, good agreement between theory and experiment was obtained. On 
average, the error with respect to the experimental data for the three substrates was around 1 $\mathrm{kcal} / \mathrm{mol}$, suggesting that the Asp99-mediated mechanism is likely to be correct.

Although the charge on an atom in a molecule is neither well-defined nor an observable quantity, atomic partial charges are readily computed and can provide valuable information about the localization of electron density in a molecular system. One method for calculating atomic charges involves performing a Natural Population Analysis (NPA), ${ }^{163,164}$ in which the molecular orbitals obtained from a quantum chemistry calculation are transformed into orthonormal, "natural" atomic orbitals. The electron populations on each atomic center are summed up, along with the nuclear charges, to yield the NPA charges. A major advantage of NPA charges over other methods, such as a Mulliken Population Analysis, ${ }^{165}$ is that they are largely unaffected by basis set dependence effects. 163

To understand how MerB achieves its catalytic power, the transition state structures for the two mechanisms were investigated using NPA charges. It was found that participation of Asp as a proton mediator enables both Cys residues to coordinate with methylmercury at the transition state (Figure 2), which is not the case for the direct protonation mechanism. Coordination of methylmercury by two Cys thiolates weakens the $\mathrm{Hg}-\mathrm{C}$ bond, increases the negative charge on the methyl carbon atom, and increases the positive charge on the catalytic proton, thus enabling the electrophilic proton on Asp99 to attack the leaving group. For the direct protonation mechanism, only one Cys is coordinated with $\mathrm{Hg}$ at the transition state, and the catalytic proton is significantly less electrophilic than in the Asp-mediated mechanism.

\section{Case study 2: Cdc25B Phosphatase}

The following is a synopsis of Parks et al. (2009) 'Mechanism of Cdc25B Phosphatase with the Small Molecule Substrate $p$-Nitrophenyl Phosphate from QM/MM-MFEP Calculations'. ${ }^{166}$ Here, the emphasis is on using experimental data from site-directed mutagenesis, kinetics measurements, $\mathrm{pH}-$ rate profiles, and isotope effects measurements in conjunction with a $\mathrm{QM} / \mathrm{MM}$ free energy 
simulation to provide insight into an enzymatic reaction mechanism.

Cdc25B is a phosphatase enzyme that dephosphorylates two residues on the Cdk2/CycA protein complex, and in so doing regulates the human cell cycle. ${ }^{167}$ In general, protein tyrosine phosphatases (PTPases) are thought to bind phosphate dianions and use a general acid to facilitate $\mathrm{P}-\mathrm{O}$ bond cleavage. ${ }^{168}$ However, Cdc25 phosphatases lack the conserved Asp residue believed to serve as the catalytic acid in other PTPases. Computer simulation may provide useful insight into how Cdc25B achieves catalysis without this seemingly important residue.

Cdc25B cleaves $\mathrm{P}-\mathrm{O}$ bonds in phosphate monoesters via two separate chemical steps (Scheme 2). In the first step, a deprotonated Cys attacks the scissile $\mathrm{P}-\mathrm{O}$ bond and forms a covalent phosphocysteine intermediate. In the second step, the phosphocysteine is hydrolyzed and inorganic phosphate is released.

The nucleophilic cysteine, Cys473 in Cdc25B, is deprotonated and has a $K_{a}$ of 5.9. ${ }^{169}$ Mutation studies showed previously that Cys473 and Arg479 are required for enzyme activity. ${ }^{170}$ Two active site residues, Glu474 and Glu478, have been considered as possible catalytic acids, but the pH-rate profiles for the Glu474Gln and Glu478Gln mutants remained unchanged relative to the wild-type enzyme, demonstrating that neither is the catalytic acid. ${ }^{171}$

The small-molecule substrate $p$-nitrophenylphosphate (pNPP) was chosen for the simulations because its dephosphorylation reaction is well characterized experimentally and its reaction with Cdc25B has been shown to proceed without acid catalysis. ${ }^{172}$ Because the formation of the phosphocysteine intermediate is known to be rate-limiting, ${ }^{171}$ only the $\mathrm{P}-\mathrm{O}$ cleavage step (Scheme 2) was considered in the calculations. The experimental $\mathrm{k}_{\mathrm{cat}}$ for $\mathrm{Cdc} 25 \mathrm{~B}$ with pNPP substrate is 0.17 $\mathrm{s}^{-1},{ }^{171}$ which corresponds to an activation free energy of $\Delta \mathrm{G}^{\ddagger}=18.5 \mathrm{kcal} / \mathrm{mol}$ at $298 \mathrm{~K}$ using TST.

The QM/MM Minimum Free Energy Path (QM/MM-MFEP) method ${ }^{122}$ was used to simulate the Cdc25B-catalyzed cleavage of the scissile $\mathrm{P}-\mathrm{O}$ bond of the pNPP substrate. Two reaction mechanisms were simulated, with the pNPP substrate being modeled as a phosphate dianion in the first mechanism and a monoanion in the second. The QM subsystem, which consisted of the sidechains of Cys473 and Arg 479, the pNPP substrate, and one water molecule, was described 
using the B3LYP/6-31G(d) level of theory. The pseudobond method ${ }^{73}$ was used to cap the free valences at the QM/MM interface.

For the first mechanism, the dianionic pNPP substrate underwent $\mathrm{P}-\mathrm{O}$ cleavage with an unexpectedly low computed activation free energy of $\sim 1 \mathrm{kcal} / \mathrm{mol}$ in which the reactant state was found to be quite high in energy and only marginally more stable that the transition state (not shown). Although a low activation free energy seems to suggest efficient catalysis, the disparity between the computed and experimental barriers suggested that the mechanism is not likely to be correct. Additionally, classical molecular dynamics simulations of the Cdc25B-pNPP dianion system revealed that the substrate did not remain bound in the active site, but dissociated due to electrostatic repulsion. Taken together, these results suggest that the dianionic form of pNPP is not in the correct ionization state to bind to $\mathrm{Cdc} 25 \mathrm{~B}$.

For the second mechanism, a free energy of activation of $\sim 23 \mathrm{kcal} / \mathrm{mol}$ was obtained, in reasonable agreement with the experimental value of $18.5 \mathrm{kcal} / \mathrm{mol}$. The calculated structure for the monoanionic pNPP substrate was characterized as a dissociative, metaphosphate with a scissile $\mathrm{P}-\mathrm{O}$ bond distance of $2.12 \AA$ and a P-S(Cys473) distance of $2.82 \AA$ (Figure 3$)$. This structure is consistent with experimental $(\mathrm{V} / \mathrm{K})$ values of ${ }^{15} \mathrm{~N}$ and ${ }^{18} \mathrm{O}_{\text {scissile, }}$, which suggested a dissociative transition state without leaving group protonation. However, if the phosphate is monoprotic, the ${ }^{18} \mathrm{O}_{\text {non-scissile }} \mathrm{KIE}$ would be $\sim 1.014$, suggesting that deprotonation of the phosphate group occurs at the transition state. The B3LYP/6-31G(d)/CHARMM22 QM/MM-MFEP calculations predicted the phosphoryl proton transfer after the transition state, in contrast with the experimental data.

Although the overall agreement between theory and experiment is good, there are important differences that may be due to the chosen level of theory, the simulation protocol, or both. Nevertheless, the QM/MM-MFEP simulations used in the study provided evidence that Cdc25 phosphatases may be unique in their preference for monoanionic rather than dianion phosphate substrates. 


\section{Case Study 3: The Restriction Enzyme, EcoRV}

The following is a synopsis of Imhof et al. (2009) 'Catalytic Mechanism of DNA Backbone Cleavage by the Restriction Enzyme EcoRV'. ${ }^{173}$ Here, the emphasis is on the exploration of many different pathways at a relatively low level of theory in QM/MM calculations. The chosen level of theory was first validated by using calculations of model systems and comparing them to high-level density functional theory results.

EcoRV is an endonuclease that cleaves the DNA backbone at a specific recognition sequence by hydrolyzing the phosphodiester bond. A magnesium cofactor is essential for enzyme activity. Extensive crystallographic studies of wild-type and various mutants of EcoRV in complex with different metal ions, and/or its substrate DNA, have led to a number of proposals of how catalysis is achieved. However, neither the precise roles of the active site residues and the metal cofactor, nor the number of metal ions required for catalysis, are clear from experimental characterization.

The two extreme cases of phosphate hydrolysis mechanisms are the dissociative and associative pathways. In a dissociative mechanism, the leaving group OR' departs before nucleophilic attack occurs, leading to the formation of a trigonal planar metaphosphate. In contrast, the associative pathway exhibits a pentavalent, trigonal bipyramidal transition state or intermediate structure, resulting from nucleophilic attack prior to $\mathrm{P}-\mathrm{O}$ bond cleavage. For the associative pathway, the seemingly clear catalytic role of the metal cofactor is the stabilization of the transition state by compensating for the accumulated negative charge of -2 (phosphate and hydroxyl), whereas the metal would seem to play a less direct role in the dissociative case.

To discern the structural and energetic differences between dissociative and associative phosphate hydrolysis mechanisms catalyzed by EcoRV, a computational study was performed by applying QM-only (AM1/d and B3LYP/6-31++ $\mathrm{G}^{* *}$ ) calculations to the uncatalyzed hydrolysis of Mg-bound dimethylphosphate, and hybrid QM/MM (AM1/d/CHARMM22) calculations to the enzymatic reaction. Model calculations demonstrated that reaction-specific AM1/d parameters developed for phosphorus ${ }^{153}$ and magnesium ${ }^{152}$ used in this study were sufficiently accurate to reproduce the reaction energetics computed using high-level DFT. 
Using a QM/MM (AM1/d/CHARMM22) approach, a network of minimum energy pathways was generated. Because a semi-empirical QM method was used, it was possible to consider a large number of possible reaction pathways. Several intermediate structures were generated by varying the $\mathrm{P}-\mathrm{O}$ distances, placing protons at different sites, and performing QM/MM geometry optimizations. The pathways for the transition between two intermediates (or intermediate and end state) were computed using the conjugate peak refinement (CPR) algorithm, ${ }^{108}$ which does not require a predefined reaction coordinate but determines a transition pathway between two given end states by a series of line maximizations and conjugate minimizations. In this manner, dissociative and associative pathways with different proton transfer steps involved were examined.

QM/MM calculations predicted that the dissociative pathway was energetically most favorable (Fig. Figure 4). The reaction is initiated by a Mg-bound water molecule (w1) leaving the metal coordination sphere and aligning with the scissile $\mathrm{P}-\mathrm{O}$ bond. Then the leaving group, which is protonated by another $\mathrm{Hg}$-activated water molecule (w2), departs. The inline-positioned water molecule transfers a proton to Asp90 and attacks the phosphorous atom. Re-protonation of the deprotonated $\mathrm{Hg}$-bound water molecule w2 is achieved by proton transfer from Asp90 to w2 via protonation of the scissile phosphate group.

The two energetically most favorable associative mechanisms differ mainly in the generation of the nucleophile. In the more likely pathway based on energetic considerations, the $\mathrm{Mg}$-bound water molecule w1 transfers a proton to Asp90. The proton is then further transferred to the phosphate group, and subsequently the nucleophile attacks, whereas the alternative is a proton relay from w1 via Lys92 to the phosphate group prior to nucleophilic attack. The decay of the associative intermediate is in both cases the departure of the leaving group, again protonated by the $\mathrm{Hg}$-activated water molecule w2.

The computation of an extensive network of reaction pathways for the hydrolysis of the phosphodiester in the DNA backbone allowed the identification of the most probable reaction mechanism, as well as possible alternative mechanisms. Moreover, inclusion of additional pathways in the network, such as the nucleophilic attack of an alternative water molecule or dissociation of 
other, non-scissile $\mathrm{P}-\mathrm{O}$ bonds in the phosphodiester, explains the preference for the actual nucleophile and the specificity for the cleavage of $\mathrm{P}-\mathrm{O} 3$ ' over the $\mathrm{P}-\mathrm{O} 5$ ' bond. The network approach further enabled the determination of steps in the reaction that are common to several mechanisms. For example, in all pathways a change of the metal coordination number from 6 to 4 takes place, explaining why larger ions such as calcium, which are unlikely to have coordination numbers below six, are catalytically inactive.

A comparison of the associative pathways in the enzyme and model systems shows similar mechanisms and similar barriers for the enzymatic and the uncatalyzed reaction. The following effects were found to contribute to catalysis in EcoRV: proper alignment of the scissile $\mathrm{P}-\mathrm{O}$ bond in the enzyme active site, activation of a nucleophilic water molecule by $\mathrm{Mg}$, participation of an Asp residue as a general base to accept a proton from the nucleophilic water molecule, and electrostatic stabilization of the transition state by the enzyme active site.

By applying a computationally less demanding SQM method, the computation of many possible pathways was affordable. Moreover, competing mechanisms were able to be explored and the preference for the native reaction was explained based on energetic considerations. The comparison with model systems served not only to validate the applied method but also to reveal that the catalytic reaction in the enzyme is qualitatively different from the one in small molecule systems.

\section{Common pitfalls}

Care must be taken to ensure that simulations of enzyme catalysis are set up and performed correctly. The following list is by no means exhaustive, but it serves to point out a few of the more common mistakes likely to be encountered in computational studies. First, detailed knowledge of the existing experimental and computational data for a given system of interested must be obtained. For example, it is essential to ensure that the protonation states of all ionizable residues are assigned correctly in accord with experimental or predicted $\mathrm{p} K_{a}$ data. Second, the chosen level of approximation must be carefully tested and validated with appropriate model calculations. This step 
should not be overlooked; a given of theory may perform well for a particular class of molecules or reactions, but may produce completely wrong results for other systems. In the quantum cluster approach, gas phase calculations are likely to yield erroneous protonation states relative to the true system. ${ }^{174}$ In other words, the electrostatic environment in the protein is most likely quite different from an isolated gas-phase system. Thus, it must be decided whether or not environmental effects should be included either explicitly or implicitly during geometry optimizations. Another common mistake involves a poorly chosen system size, either in the cluster approach or in partitioning of the QM subsystem in QM/MM calculations. Neglect of key residues or groups will undoubtedly result in significant errors.

An inadequate level of quantum mechanical theory will result in qualitatively or quantitatively incorrect results. For example, standard SQM methods generally have difficulty in describing transition metals, hydrogen bonds, and dispersion interactions ${ }^{24}$ unless corrections are applied ${ }^{155,156}$ and DFT may perform poorly in computing reaction barriers for chemical systems that exhibit significant static correlation effects. ${ }^{175}$ In QM/MM simulations employing potential energies (as opposed to free energies) it is quite common for structures to become trapped in local minima, which will result in discontinuous reaction paths. One remedy is to "drive" the reaction coordinate forward and backward between the reactant and product states multiple times until a smooth, continous reaction path is obtained. However, this approach can be quite time-consuming, particularly when a computationally intensive quantum chemical method is used. It is also possible to obtain activation energies that differ by many $\mathrm{kcal} / \mathrm{mol}$ when potential energy surfaces are generated using different initial enzyme configurations, ${ }^{176,177}$ and methods have been developed to circumvent these difficulties. ${ }^{96,122}$ In free energy simulations, insufficient sampling will adversely affect the quality of the results. Thus adequate sampling is required, although it remains particularly challenging for QM/MM MD simulations because of the computational cost. ${ }^{65}$ 


\section{Summary and outlook}

Simulations of enzyme catalysis are not black-box calculations. Expertise is required to understand the detailed biochemistry involved, as well as the underlying methods and approximations used. Potential areas for progress in simulating enzyme reactions include developing more accurate and efficient quantum chemical methods such as improved DFT functionals ${ }^{175,178}$ and semi-empirical methods, polarizable force fields, ${ }^{17}$ robust methods for determining reaction paths, and improved descriptions of electrostatics and free energy sampling methods. ${ }^{174}$

Furthermore, chemomechanical coupling, ${ }^{179-183}$ in which a chemical reaction precedes or is the result of a mechanical (conformational) change, will be a fruitful field of investigation. Dynamic effects on catalysis also remain a topic of intense debate. ${ }^{184-192}$ Computer simulation will continue to play an increasingly important role in understanding how enzymes achieve their tremendous catalytic power.

\section{Acknowledgement}

This research was funded by the Environmental Remediation Sciences Program (ERSP) and the BioEnergy Science Center, which are supported by the U.S. Department of Energy Office of Science, Office of Biological and Environmental Research. The BioEnergy Science Center is a U.S. Department of Energy Bioenergy Research Center. Oak Ridge National Laboratory is managed by UT-Battelle, LLC, for the U.S. Department of Energy under contract DE-AC05-00OR22725. 


\section{References}

(1) Fersht, A. Structure and Mechanism in Protein Science. A Guide to Enzyme Catalysis and Protein Folding; W. H. Freeman and Co.: New York, 1999.

(2) Silverman, R. The Organic Chemistry of Enzyme-Catalyzed Reactions; CA Academic Press: San Diego, CA, 2000.

(3) Radzicka, A.; Wolfenden, R. Science 1995, 267, 90-93.

(4) Wolfenden, R.; Snider, M. J. Acc. Chem. Res. 2001, 34, 938-945.

(5) Wolfenden, R. Biophys. Chem. 2003, 105, 559-572.

(6) Eyring, H. J. Chem. Phys. 1935, 3, 107-115.

(7) Siegbahn, P. E. M.; Borowski, T. Acc. Chem. Res. 2006, 39, 729-738.

(8) Cleland, W. W. Arch. Biochem. Biophys. 2005, 433, 2-12.

(9) Schramm, V. L. Ann. Rev. Biochem. 1998, 67, 693-720.

(10) Hengge, A. C. Acc. Chem. Res. 2002, 35, 105-112.

(11) Cramer, C. J. Essentials of Computational Chemistry, Second Edition: Theories and Models; John Wiley and Sons, Ltd.: West Sussex, England, 2004.

(12) Ponder, J. W.; Case, D. A. Force field methods for protein simulations. Advances in Protein Chemistry, 2003; pp 27-86.

(13) Cornell, W.; Cieplak, P.; Bayly, C.; Gould, I.; Merz Jr., K. M.; Ferguson, D. M.; Spellmeyer, D.; Fox, T.; Caldwell, J.; Kollmann, P. J. Am. Chem. Soc. 1995, 117, 5179_ 5197.

(14) Brooks, B. R. et al. J. Comput. Chem. 2009, 30, 1545-1615. 
(15) Oostenbrink, C.; Villa, A.; Mark, A. E.; van Gunsteren, W. F. J. Comput. Chem. 2004, 25, $1656-1676$.

(16) Jorgensen, W. L.; Maxwell, D.; Tirado-Rives, J. J. Am. Chem. Soc. 1996, 118, 11225-11236.

(17) J. W. Ponder and C. Wu and P. Ren and V. S. Pande and J. D. Chodera and M. J. Schnieders and I. Haque and D. L. Mobley and D. S. Lambrecht and R. A. DiStasio Jr. and M. HeadGordon and G. N. I. Clark and M. E. Johnson and T. Head-Gordon, J. Phys. Chem. B 2010, $114,2549-2564$.

(18) Gresh, N.; Cisneros, G. A.; Darden, T. A.; Piquemal, J.-P. J. Chem. Theory Comput. 2007, 3, 1960-1986.

(19) Hehre, W. J.; Radom, L.; Schleyer, P. V.; Pople, J. Ab Initio Molecular Orbital Theory; John Wiley and Sons, Inc.: USA, 1986.

(20) Szabo, A.; Ostlund, N. S. Modern Quantum Chemistry; Dover Publications, Inc.: Mineola, New York, 1996.

(21) Parr, R. G.; Yang, W. Density Functional Theory of Atoms and Molecules; Oxford University Press: New York, 1989.

(22) Koch, W.; Holthausen, M. C. A Chemist's Guide to Density Functional Theory, Second Edition; Wiley-VCH: Weinheim, Germany, 2001.

(23) Thiel, W. Adv. Chem. Phys. 1996, 93, 703-757.

(24) Clark, T. J. Mol. Struct. (Theochem) 2000, 530, 1-10.

(25) Elstner, M.; Porezag, D.; Jungnickel, G.; Elsner, J.; Haugk, M.; Frauenheim, T.; Suhai, S.; Seifert, G. Phys. Rev. B 1998, 58, 7260-7268.

(26) Siegbahn, P. E. M.; Himo, F. J. Biol. Inorg. Chem. 2009, 14, 643-651.

(27) Siegbahn, P.; Blomberg, M. Chem. Rev. 2000, 100, 421-437. 
(28) Blomberg, M. R. A.; Siegbahn, P. E. M. J. Phys. Chem. B 2001, 105, 9375-9386.

(29) Siegbahn, P. E. M. Curr. Opin. Chem. Bio. 2002, 6, 227-235.

(30) Himo, F.; Siegbahn, P. E. M. Chem. Rev. 2003, 103, 2421-2456.

(31) Lovell, T.; Himo, F.; Han, W.-G.; Noodleman, L. Coord. Chem. Rev. 2003, 238-239, 211232.

(32) Noodleman, L.; Lovell, T.; Han, W.-G.; Li, J.; Himo, F. Chem. Rev. 2004, 104, 459-508.

(33) Himo, F. Theor. Chem. Acc. 2006, 116, 232-240.

(34) Siegbahn, P. E. M.; Tye, J. W.; Hall, M. B. Chem. Rev. 2007, 107, 4414-4435.

(35) Ramos, M. J.; Fernandes, P. A. Acc. Chem. Res. 2009, 41, 689-698.

(36) Becke, A. D. Phys. Rev. A 1988, 38, 3098-3100.

(37) Becke, A. D. J. Chem. Phys. 1993, 98, 5648-5652.

(38) Lee, C.; Yang, W.; Parr, R. G. Phys. Rev. B 1988, 37, 785-789.

(39) Tomasi, J.; Mennucci, B.; Cammi, R. Chem. Rev. 2005, 105, 2999-3093.

(40) Klamt, A.; Schüürmann, G. J. Chem. Soc. Perkins Trans. 1993, 2, 799-805.

(41) Andzelm, J.; Kölmel, C.; Klamt, A. J. Chem. Phys. 1995, 103, 9312-9320.

(42) Barone, V.; Cossi, M. J. Phys. Chem. A 1998, 102, 1995-2001.

(43) Cossi, M.; Rega, N.; Scalmani, G.; Barone, V. J. Comput. Chem. 2003, 24, 669-681.

(44) York, D. M.; Karplus, M. J. Phys. Chem. A 1999, 103, 11060-11079.

(45) Boese, A. D.; Martin, J. M. L. J. Chem. Phys. 2004, 1121, 3405-3416.

(46) Zhao, Y.; Pu, J.; Lynch, B. J.; Truhlar, D. G. Phys. Chem. Chem. Phys. 2004, 6, 673-676. 
(47) Zhao, Y.; González-García, N.; Truhlar, D. G. J. Phys. Chem. A 2005, 109, 2012-2018.

(48) Li, J.; Fisher, C. L.; Konecny, R.; Bashford, D.; Noodleman, L. Inorg. Chem. 1999, 38, 929-939.

(49) Warshel, A.; Levitt, M. J. Mol. Bio. 1976, 103, 227-249.

(50) Field, M. J.; Bash, P. A.; Karplus, M. J. Comput. Chem. 1990, 11, 700-733.

(51) Gao, J. Methods and applications of combined quantum mechanical and molecular mechanical potentials. Reviews in Computational Chemistry, 1995; pp 119-186.

(52) Friesner, R. A.; Guallar, V. Ann. Rev. Phys. Chem. 2005, 56, 389-427.

(53) Murphy, R. B.; Phillipp, D. M.; Friesner, R. A. J. Comput. Chem. 2000, 21, 1442-1457.

(54) Gogonea, V. Internet Electron. J. Mol. Des. 2003, 1, 173-184.

(55) Gao, J.; Truhlar, D. G. Ann. Rev. Phys. Chem. 2002, 53, 467-505.

(56) Garcia-Viloca, M.; Gao, J.; Karplus, M.; Truhlar, D. G. Science 2004, 303, 186-195.

(57) Åqvist, J.; Warshel, A. Chem. Rev. 1993, 93, 2523-2544.

(58) Villà, J.; Warshel, A. J. Phys. Chem. B 2001, 105, 7887-7907.

(59) Warshel, A. Annu. Rev. Biophys. Biomol. Struct. 2003, 32, 425-443.

(60) Lin, H.; Truhlar, D. G. Theor. Chem. Acc. 2007, 117, 185-199.

(61) Field, M. J. A Practical Introduction to the Simulation of Molecular Systems, Second Edition; Cambridge University Press: Cambridge, U.K., 2007.

(62) Hu, H.; Yang, W. Ann. Rev. Phys. Chem. 2008, 59, 573-601.

(63) Acevedo, O.; Jorgensen, W. L. Acc. Chem. Res. 2010, 43, 142-151. 
(64) Senn, H. M.; Thiel, W. QM/MM Methods for Biological Systems. In Atomistic Approaches in Modern Biology: From Quantum Chemistry to Molecular Simulations; Springer-Verlag Berlin Heidelberg, 2007; Vol. 268, pp 173-290.

(65) Senn, H. M.; Thiel, W. Angew. Chem. Int. Ed. 2009, 48, 1198-1229.

(66) Antes, I.; Thiel, W. J. Phys. Chem. A 1999, 103, 9290-9295.

(67) Théry, V.; Rinaldi, D.; Rivail, J.-L.; Maigret, B.; Ferenczy, G. G. J. Comput. Chem. 1994, $15,269-282$.

(68) Reuter, N.; Dejaegere, A.; Maigret, B.; Karplus, M. J. Phys. Chem. A 2000, 104, 1720-1735.

(69) Gao, J.; Amara, P.; Alhambra, C.; Field, M. J. J. Phys. Chem. A 1998, 102, 4714-4721.

(70) Pu, J.; Gao, J.; Truhlar, D. G. J. Phys. Chem. A 2004, 108, 5454-5463.

(71) Pu, J.; Gao, J.; Truhlar, D. G. J. Phys. Chem. A 2004, 108, 632-650.

(72) Zhang, Y.; Lee, T.-S.; Yang, W. J. Chem. Phys. 1999, 110, 46-54.

(73) Zhang, Y. J. Chem. Phys. 2005, 122, 024114.

(74) Zhang, Y. J. Chem. Phys. 2006, 116, 43-50.

(75) Parks, J. M.; Hu, H.; Cohen, A. J.; Yang, W. J. Chem. Phys. 2008, 129, 154106.

(76) Car, R.; Parrinello, M. Phys. Rev. Lett. 1985, 55, 2471-2474.

(77) Jorgensen, W. L.; Chandrasekhar, J.; Madura, J. D.; Impey, R. W.; Klein, M. L. J. Chem. Phys. 1983, 79, 926-935.

(78) Berendsen, H. J. C.; Postma, J. P. M.; van Gunsteren, W. F.; Hermans, J. Intermolecular Forces, 1981; pp 331-342.

(79) Berendsen, H. J. C.; Grigera, J. R.; Straatsma, T. P. J. Phys. Chem. 1987, 91, 6269-6271. 
(80) Darden, T.; York, D.; Pedersen, L. J. Chem. Phys. 1993, 98, 10089-10092.

(81) Nam, K.; York, D. M. J. Chem. Theory Comput. 2005, 1, 2-13.

(82) Walker, R. C.; Crowley, M. F.; Case, D. A. J. Comput. Chem. 2008, 29, 1019-1031.

(83) Berkowitz, M.; McCammon, J. A. Chem. Phys. Lett. 1982, 90, 215-217.

(84) Brooks III, C. L.; Karplus, M. J. Chem. Phys. 1983, 79, 6312-6325.

(85) Brooks III, C. L.; Brünger, A.; Karplus, M. Biopolymers 1985, 24, 843-865.

(86) Im, W.; Berneche, S.; Roux, B. J. Chem. Phys. 2001, 114, 2924-2937.

(87) Schaefer, P.; Riccardi, D.; Cui, Q. J. Chem. Phys. 2005, 123, 014905.

(88) Riccardi, D.; Cui, Q. J. Phys. Chem. A 2007, 111, 5703-5711.

(89) Benighaus, T.; Thiel, W. J. Chem. Theory Comput. 2008, 4, 1600-1609.

(90) Benighaus, T.; Thiel, W. J. Chem. Theory Comput. 2009, 5, 3114-3128.

(91) Schwarzl, S. M.; Huang, D. Z.; Smith, J. C. J. Comput. Chem. 2005, 26, 1359-1371.

(92) Simonson, T.; Archontis, G.; Karplus, M. J. Phys. Chem. B 1997, 101, 8349-8362.

(93) Dinner, A.; Lopez, X.; Karplus, M. Theor. Chem. Acc. 2003, 109, 118-124.

(94) Gregersen, B. A.; York, D. M. J. Phys. Chem. B 2005, 109, 536-556.

(95) Gregersen, B. A.; York, D. M. J. Comput. Chem. 2006, 27, 103-115.

(96) Rosta, E.; Haranczyk, M.; Chu, Z. T.; Warshel, A. J. Phys. Chem. B 2008, 112, 5680-5692.

(97) Kamerlin, S. C. L.; Haranczyk, M.; Warshel, A. J. Phys. Chem. B 2009, 113, 1253-1272.

(98) Klinman, J. P. Trends Biochem. Sci. 1989, 14, 368-373.

(99) Hwang, J.-K.; Warshel, A. J. Am. Chem. Soc. 1996, 118, 11745-11751. 
(100) Alhambra, C.; Corchado, J.; Sánchez, M. L.; Garcia-Viloca, M.; Gao, J.; Truhlar, D. G. J. Phys. Chem. B 2001, 105, 11326-11340.

(101) Truhlar, D.; Gao, J.; Alhambra, C.; Garcia-Viloca, M.; Corchado, J.; Sanchez, M.; Villa, J. Acc. Chem. Res. 2002, 35, 341-349.

(102) Billeter, S. R.; Webb, S. P.; Iordanov, T.; Agarwal, P. K.; Hammes-Schiffer, S. J. Am. Chem. Soc. 2001, 114, 6925-6936.

(103) Hammes-Schiffer, S. Acc. Chem. Res. 2006, 39, 93-100.

(104) Bondar, A. N.; Elstner, M.; Fischer, S.; Smith, J. C.; Suhai, S. Phase Transit. 2004, 77, $47-52$.

(105) Elber, R.; Karplus, M. Chem. Phys. Lett. 1987, 139, 375.

(106) Czerminski, R.; Elber, R. Int. J. Quant. Chem. 1990, 24, 167.

(107) Jónsson, H.; Mills, G.; Jacobsen, K. W. Nudged Elastic Band Method. In Classical and Quantum Dynamics in Condensed Phase Simulations; Berne, B. J., Ciccotti, G., Coker, D. F., Eds.; World Scientific: Singapore, 1998; pp 387-404.

(108) Fischer, S.; Karplus, M. Chem. Phys. Lett. 1992, 194, 252-261.

(109) Burger, S. K.; Yang, W. J. Chem. Phys. 2006, 124, 054109.

(110) Wales, D. J. Mol. Phys. 2002, 100, 3285-3305.

(111) Noé, F.; Krachtus, D.; Smith, J. C.; Fischer, S. J. Chem. Theory Comput. 2006, 2, 840-857.

(112) Hamelberg, D.; J, J. M.; McCammon, J. J. Chem. Phys. 2004, 120, 11919-11929.

(113) Amadei, A.; Linssen, A. B. M.; Berendsen, H. J. C. Proteins: Struct. Func. Genet. 2004, $17,412-425$. 
(114) Kästner, J.; Senn, H. M.; Thiel, S.; Otte, N.; Thiel, W. J. Chem. Theory Comput. 2006, 2, 452.

(115) Ytreberg, F. M.; Swendsen, R. H.; Zuckerman, D. M. J. Chem. Phys. 2006, 125, 184114.

(116) Torrie, G.; Valleau, J. J. Comput. Phys. 1977, 23, 187-199.

(117) Kumar, S.; Bouzida, D.; Swendsen, R. H.; Kollman, P. A.; Rosenberg, J. M. J. Comput. Chem. 1992, 13, 1011-1021.

(118) Michael R. Shirts and John D. Chodera, J. Chem. Phys. 2008, 129, 124105.

(119) Bartels, C.; Karplus, M. Journal of Computational Chemistry 1997, 18, 1450-1462.

(120) Hooft, R.; van Eijck, B.; Kroon, J. J. Chem. Phys. 1992, 97, 6690.

(121) Zwanzig, R. W. J. Chem. Phys. 1954, 22, 1420-1426.

(122) Hu, H.; Lu, Z.; Parks, J. M.; Burger, S. K.; Yang, W. J. Chem. Phys. 2008, 128, 034105.

(123) Zhang, Y.; Liu, H.; Yang, W. J. Chem. Phys. 2000, 112, 3484-3492.

(124) Liu, H.; Zhang, Y.; Yang, W. T. J. Am. Chem. Soc. 2000, 122, 6560-6570.

(125) Cisneros, G. A.; Liu, H.; Zhang, Y.; Yang, W. J. Am. Chem. Soc. 2003, 125, 10384-10393.

(126) Rod, T. H.; Rydberg, P.; Ryde, U. J. Chem. Phys. 2006, 124, 174503.

(127) Hu, H.; Lu, Z.; Yang, W. J. Chem. Theory Comput. 2007, 3, 390-406.

(128) Hu, H.; Yang, W. J. Mol. Struct. (Theochem) 2009, 898, 17-30.

(129) Huber, T.; Torda, A. E.; van Gunsteren, W. F. J. Am. Chem. Soc. 1994, 131, 18147-18152.

(130) Grubmüller, H. Phys. Rev. E 1995, 52, 2893-2906.

(131) Laio, A.; Parinello, M. Proc. Natl. Acad. Sci. U.S.A. 2002, 99, 12562-12566. 
(132) Laio, A.; Rodriguez-Fortea, A.; Gervasio, L.; Ceccarelli, M.; Parinello, M. J. Phys. Chem. B 2005, 109, 6714-6721.

(133) Ensing, B.; de Vivo, M.; Liu, Z.; Moore, P.; Klein, M. L. Acc. Chem. Res. 2006, 39, 73-81.

(134) Bolhuis, P.; Chandler, D.; Dellago, C.; Geissler, P. Annu. Rec. Phys. Chem. 2002, 53, 291318.

(135) Dellago, C.; Bolhuis, P.; Chandler, D. J. Chem. Phys. 1998, 108, 9236-9245.

(136) Dewar, M. J. S.; Zoebisch, E. G.; Healy, E. F.; Stewart, J. J. P. J. Am. Chem. Soc. 1985, 107, 3902-3909.

(137) Kolb, M.; Thiel, W. J. Comput. Chem. 1993, 14, 775-789.

(138) Thiel, W.; Weber, W. Theor. Chem. Acc. 2000, 103, 495-506.

(139) Weber, W. Ph.D. thesis, University of Zurich: Zurich, Switzerland, 1996.

(140) Scholten, M. Ph.D. thesis, University of Düsseldorf: Düsseldorf, Germany, 2003.

(141) Stewart, J. J. P. J. Comput. Chem. 1989, 10, 209-220.

(142) Stewart, J. J. P. J. Mol. Model. 2007, 13, 1173-1213.

(143) Repasky, M. P.; Chandrasekhar, J.; Jorgensen, W. L. J. Comput. Chem. 2002, 23, 16011622.

(144) Tubert-Brohman, I.; Guimaraes, C.; Repasky, M.; Jorgensen, W. J. Comput. Chem. 2004, $25,138-150$.

(145) Tubert-Brohman, I.; Guimaraes, C.; Jorgensen, W. J. Chem. Theory Comput. 2005, 1, 817823.

(146) Elstner, M.; Porezag, D.; Jungnickel, G.; Elsner, J.; Haugk, M.; Frauenheim, T.; Suhai, S.; Seifert, G. Phys. Rev. B 2003, 58, 7260-7268. 
(147) Elstner, M.; Frauenheim, T.; Suhai, S. J. Mol. Struct. Theochem 2003, 632, 29-41.

(148) Sattelmeyer, K. W.; Tirado-Rives, J.; Jorgensen, W. L. J. Phys. Chem. A 2006, 110, 1355113559.

(149) Otte, N.; Scholten, M.; Thiel, W. J. Phys. Chem. A 2007, 111, 5751-5755.

(150) Tirado-Rives, J.; Jorgensen, W. L. J. Chem. Theory Comput. 2008, 4, 297-306.

(151) Rossi, I.; Truhlar, D. G. Chem. Phys. Lett. 1995, 233, 231-236.

(152) Imhof, P.; Noé, F.; Fischer, S.; Smith, J. C. J. Chem. Theory Comput. 2006, 2, 1050-1056.

(153) Nam, K.; Cui, Q.; Gao, J.; York, D. M. J. Chem. Theory Comput. 2007, 3, 486-504.

(154) Arantes, G. M. Phys. Chem. Chem. Phys. 2006, 8, 347-353.

(155) ̌ezáč, J.; Fanfrlík, J.; Salahub, D.; Hobza, P. J. Chem. Theory Comput. 2009, 5, 1749-1760.

(156) Korth, M.; Pitoňák, M.; Řezáč, J.; Hobza, P. J. Chem. Theory Comput. 2010, 6, 344-352.

(157) Yang, Y.; Yu, H.; York, D. M.; Cui, Q.; Elstner, M. J. Phys. Chem. A 2007, 111, 1086110873.

(158) Yang, Y.; Yu, H.; York, D.; Elstner, M.; Cui, Q. J. Chem. Theory Comput. 2008, 4, 20672084.

(159) Claeyssens, F.; Harvey, J. N.; Manby, F. R.; Mata, R. A.; Mulholland, A. J.; Ranaghan, K. E.; Schütz, M.; Thiel, S.; Thiel, W.; Werner, H.-J. Angew. Chem. Int. Ed. 2006, 45, 6856-6859.

(160) Parks, J. M.; Guo, H.; Momany, C.; Liang, L.; Miller, S. M.; Summers, A. O.; Smith, J. C. J. Am. Chem. Soc. 2009, 131, 13278-13285.

(161) Pitts, K. E.; Summers, A. O. Biochemistry 2002, 42, 10287-10296.

(162) Begley, T.; Walts, A.; Walsh, C. Biochemistry 1986, 25, 7192-7200. 
(163) Reed, A.; Weinstock, R. B.; Weinhold, F. J. Chem. Phys. 1985, 83, 735-746.

(164) Reed, A.; Curtiss, L.; Weinhold, F. Chem. Rev. 1988, 88, 899-926.

(165) Mulliken, R. J. Chem. Phys. 1955, 23, 1833-1840.

(166) Parks, J. M.; Hu, H.; Rudolph, J.; Yang, W. J. Phys. Chem. B 2009, 113, 5217-5224.

(167) Rudolph, J. Biochemistry 2007, 46, 3595-3604.

(168) Jackson, M. D.; Denu, J. M. Chem. Rev. 2001, 101, 2313-2340.

(169) Rudolph, J. Biochemistry 2002, 41, 14613-14623.

(170) Xu, X.; Burke, S. P. J. Biol. Chem. 1996, 271, 5118-5124.

(171) Chen, W.; Wilborn, M.; Rudolph, J. Biochemistry 2000, 39, 10781-10789.

(172) McCain, D. F.; Catrina, I. E.; Hengge, A. C.; Zhang, Z.-Y. J. Biol. Chem. 2002, 277, 1119011200.

(173) Imhof, P.; Fischer, S.; Smith, J. C. Biochemistry 2009, 48, 9061-9075.

(174) Kamerlin, S. C. L.; Haranczyk, M.; Warshel, A. J. Phys. Chem. B 2009, 113, 1253-1272.

(175) Cohen, A. J.; Mori-Sánchez, P.; Yang, W. Science 2008, 321, 792-794.

(176) Klähn, M.; Braun-Sand, S.; Rosta, E.; Warshel, A. J. Phys. Chem. B 2005, 109, 1564515650.

(177) Zhang, Y.; Kua, J.; McCammon, J. A. J. Phys. Chem. B 2003, 107, 4459-4463.

(178) Cramer, C. J.; Truhlar, D. G. Phys. Chem. Chem. Phys. 2009, 11, 10757-10816.

(179) Geeves, M.; Holmes, K. Ann. Rev. Biochem. 1999, 68, 687-728.

(180) Holmes, K.; Geeves, M. Phil. Trans. R. Soc. Lond. B 2000, 355, 419-431. 
(181) Geeves, M.; Holmes, K. Adv. Prot. Chem. 2005, 71, 161-193.

(182) Schwarzl, S.; Smith, J.; Fischer, S. Biochemistry 2006, 45, 5830-5847.

(183) Mesentean, S.; Koppole, S.; Smith, J. C.; Fischer, S. J. Mol. Bio. 2007, 367, 591-602.

(184) Hammes, G. Biochemistry 2002, 41, 8221-8228.

(185) Benkovic, S.; Hammes-Schiffer, S. Science 2003, 301, 1196-1202.

(186) Eisenmesser, E.; Millet, O.; Labeikovsky, W.; Korzhnev, D.; Wolf-Watz, M.; Bosco, D.; Skalicky, J.; Kay, L.; Kern, D. Nature 2005, 438, 117-121.

(187) Boehr, D. D.; McElheny, D.; Dyson, H. J.; Wright, P. E. Science 2006, 313, 1638-1642.

(188) M., O. M. H.; Parson, W. W.; Warshel, A. Chem. Rev. 2006, 106, 1737-1756.

(189) Henzler-Wildman, K.; Thai, V.; Lei, M.; Ott, M.; Wolf-Watz, M.; Fenn, T.; Pozharski, E.; Wilson, M. A.; Petsko, G. A.; Karplus, M.; Hübner, C. G.; Kern, D. Nature 2007, 450, 838-844.

(190) Henzler-Wildman, K.; Lei, M.; Thai, V.; Kerns, S.; Karplus, M.; Kern, D. Nature 2007, 450, 913-916.

(191) Pisliakov, A. V.; Cao, J.; Kamerlin, S. C. L.; Warshel, A. Proc. Natl. Acad. Sci. U.S.A. 2009, $106,17359-17364$.

(192) Kamerlin, S. C. L.; Warshel, A. Proteins: Struct. Func. Bioinf. 2010, 78, 1339-1375. 


$$
[\mathrm{R}-\mathrm{Hg}(\mathrm{II})]^{+} \stackrel{\mathrm{MerB}}{\longrightarrow}[\mathrm{Hg}(\mathrm{II})]^{2+}+\mathrm{R}-\mathrm{H}
$$

Scheme 1: Reaction catalyzed by MerB ( $\mathrm{R}=$ alkyl or aryl). Reprinted with permission from $J$. Am. Chem. Soc. 131, 13278-13285 (2009). Copyright 2009 American Chemical Society. 


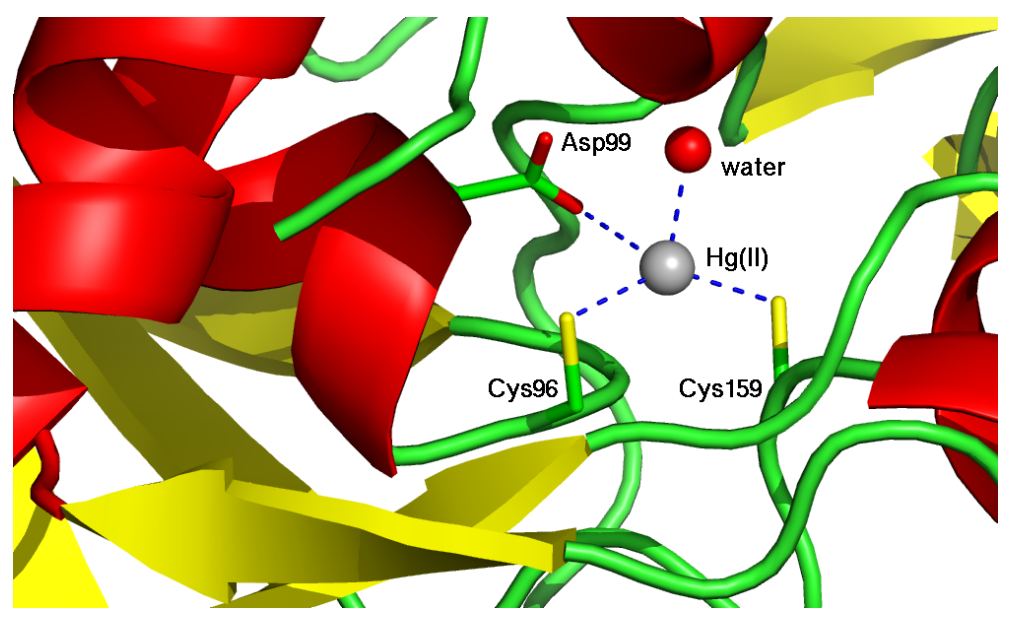

Figure 1: Active site of MerB (PDB ID 3F2F). Reprinted with permission from J. Am. Chem. Soc., 131, 13278-13285 (2009). Copyright 2009 American Chemical Society. 


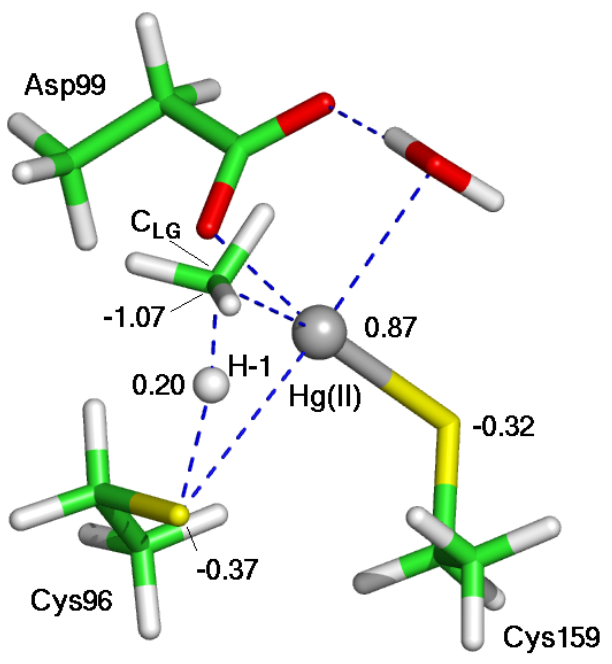

(a) Mechanism I

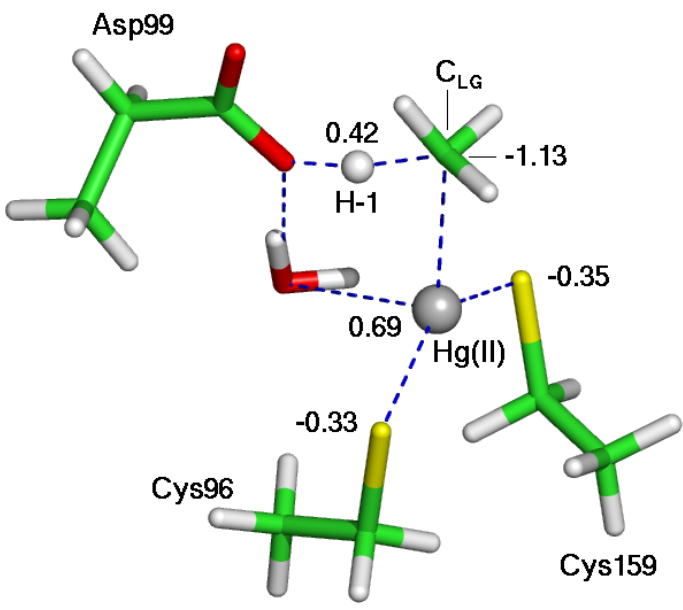

(b) Mechanism II

Figure 2: Transition state structures and NPA charges for Mechanisms 1 and 2. The activation energies for the two mechanisms are 35.9 and $20.4 \mathrm{kcal} / \mathrm{mol}$, respectively, compared with the experimentally measured value of $20.1 \mathrm{kcal} / \mathrm{mol}$. Reprinted with permission from $J$. Am. Chem. Soc., 131, 13278-13285 (2009). Copyright 2009 American Chemical Society. 

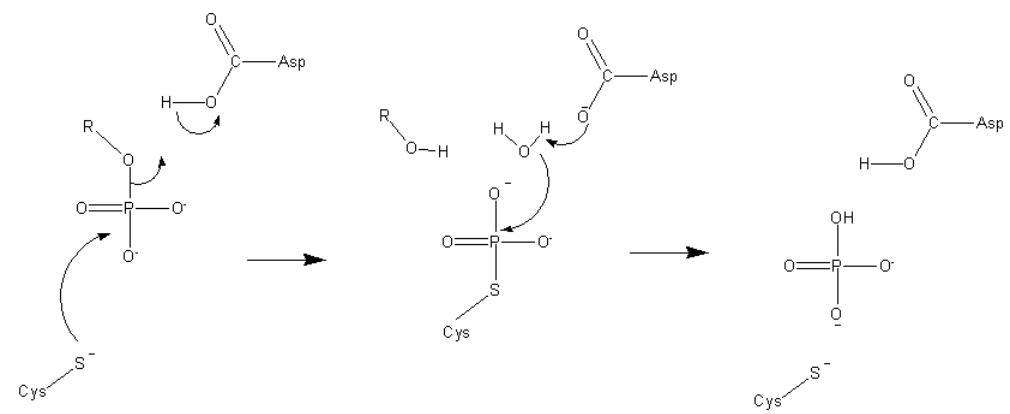

Scheme 2: Reaction catalyzed by protein tyrosine phosphatases. Reprinted with permission from J. Phys. Chem. B, 113, 5217-5224 (2009). Copyright 2009 American Chemical Society. 


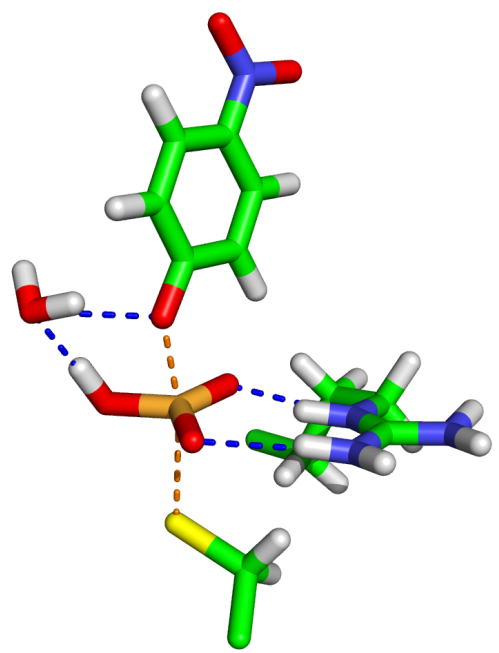

Figure 3: Computed transition state structure for the hydrolysis of the pNPP monoanion by Cdc25B. Selected bond distances (in $\AA$ ) are labeled. Only the atoms in the QM subsystem are shown for clarity. Reprinted with permission from J. Phys. Chem. B, 113, 5217-5224 (2009). Copyright 2009 American Chemical Society. 


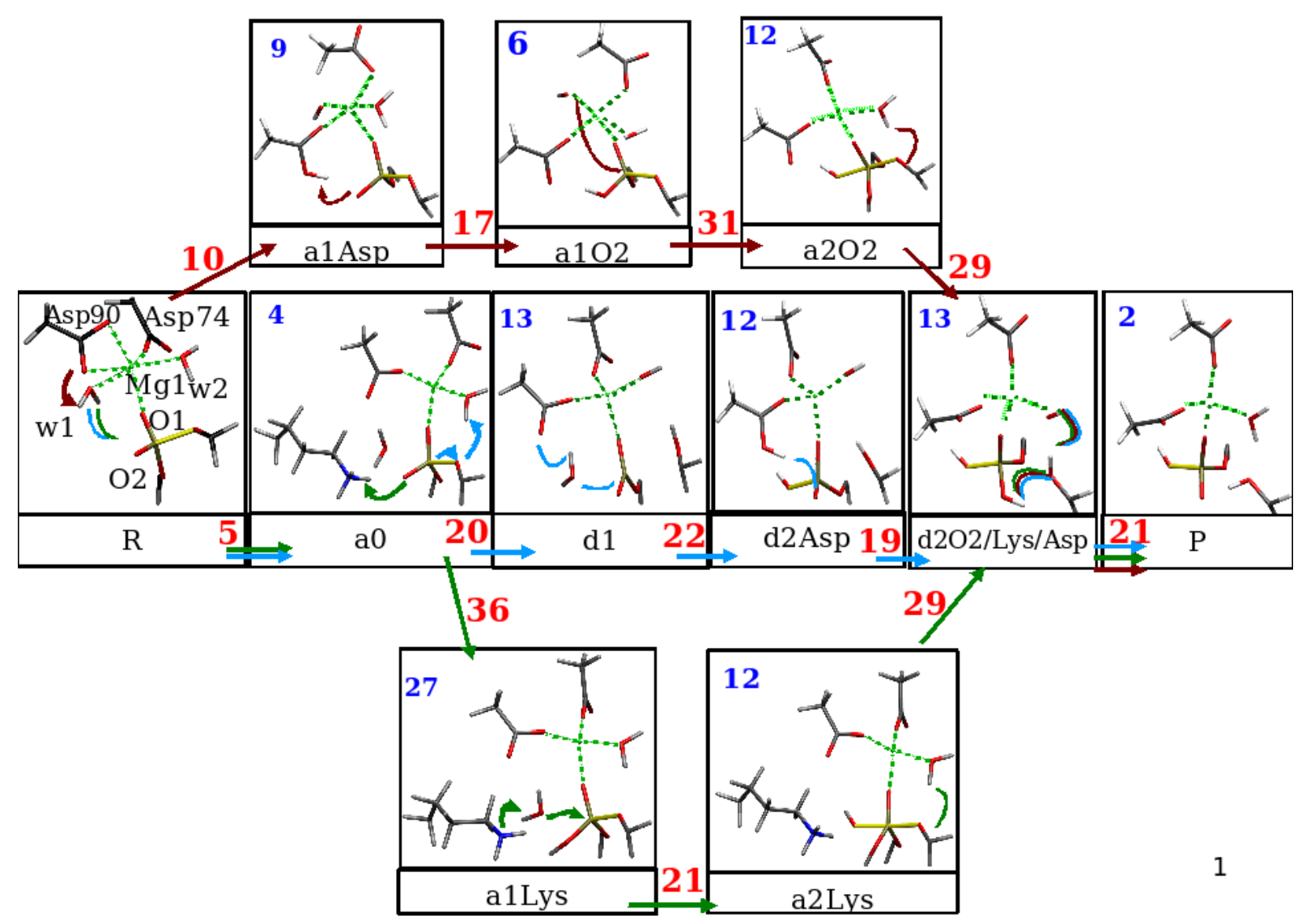

Figure 4: Energetically most favorable pathways for the phosphate hydrolysis reaction catalyzed by the restriction enzyme EcoRV. Energies (in $\mathrm{kcal} / \mathrm{mol}$ ) of intermediate states are given in blue and transition state energies are shown in red. The three different pathways are distinguished by colored arrows, and the corresponding atom movements are indicated with arrows of the respective color. The most likely pathway is the dissociative route $\mathrm{R}-\mathrm{a} 0-\mathrm{d} 1-\mathrm{d} 2 \mathrm{Asp}-\mathrm{d} 2 \mathrm{O} 2-\mathrm{P}$, labeled in light blue and shown in the center. Reprinted with permission from Biochem., 48, 9061-9075 (2009). Copyright 2009 American Chemical Society. 